\title{
Game Technology for Training and Education
}

\author{
Rafael Bidarra ${ }^{1}$ and Xiaopeng Zhang $^{2}$ \\ ${ }^{1}$ Computer Graphics Group, Faculty of Electrical Engineering, Mathematics and Computer Science (EEMCS), \\ Delft University of Technology, 2628 CD Delft, The Netherlands \\ ${ }^{2}$ LIAMA-NLPR, Institute of Automation, Chinese Academy of Sciences, Beijing 100190, China
}

Correspondence should be addressed to Rafael Bidarra, r.bidarra@ewi.tudelft.nl

Received 23 April 2009; Accepted 23 April 2009

Copyright ( $\odot 2009$ R. Bidarra and X. Zhang. This is an open access article distributed under the Creative Commons Attribution License, which permits unrestricted use, distribution, and reproduction in any medium, provided the original work is properly cited.

Serious games are being more and more deployed in such diverse areas as public awareness, military training, and higher education. One of the driving forces behind this stems from the rapidly growing availability of game technologies, providing not only better, faster, and more realistic graphics, physics, and animations, but above all making "the language" of game development accessible to increasingly more people. The articles in this special issue are a rich sampling of how game technology can be creatively put to service within games for training and education purposes.

The first two articles are focused on simulation applications. In "SIDH - a game-based architecture for a training simulator," Backlund et al. extend the notion of gamebased simulation proposing an architecture for a professional firefighter training simulator that incorporates novel visualization and interaction modes. The serious game, developed in cooperation with the government agency responsible for the training of fire and rescue personnel, is a good example of how game technology helps making the delicate combination of engaging level design and carefully tuned learning objectives. In "Experiential learning in vehicle dynamics education via motion simulation and interactive gaming," Hulme et al. present an original methodology developed within their vehicle dynamics curriculum, incorporating a combination of driving simulation, motion simulation, and educational practices into a game-based simulation framework. The paper also includes a comprehensive evaluation of their approach in two practical, educational scenarios.

The next two articles describe specific applications of game technology to serious games aimed at computer science education. In "An application of game development framework in higher education," Wang et al. present their experiences with an XNA-based game project for teaching software architecture. The paper also includes a brief survey and evaluation of game frameworks currently available for these purposes. On the other hand, in "Towards a serious game to help students learn computer programming," Muratet et al. describe the design and development of a strategy game aimed at strengthening novice programming skills, including an interesting discussion on the mapping of learning objectives into the game play.

Finally, in "Venation skeleton-based modeling plant leaf wilting," Lu et al. present an original method for simulating and visualizing leaf wilting, useful in the domain of biology. Interestingly, their technique can be applied both in a didactic setting and within any graphics framework requiring realistic visualization of natural phenomena.

We believe that serious games will continue to foster the development of new game technologies, and, conversely, combining and deploying existing game technology in novel ways will likely extend and amplify the impact and innovation achieved by these games in our society.

Rafael Bidarra Xiaopeng Zhang 

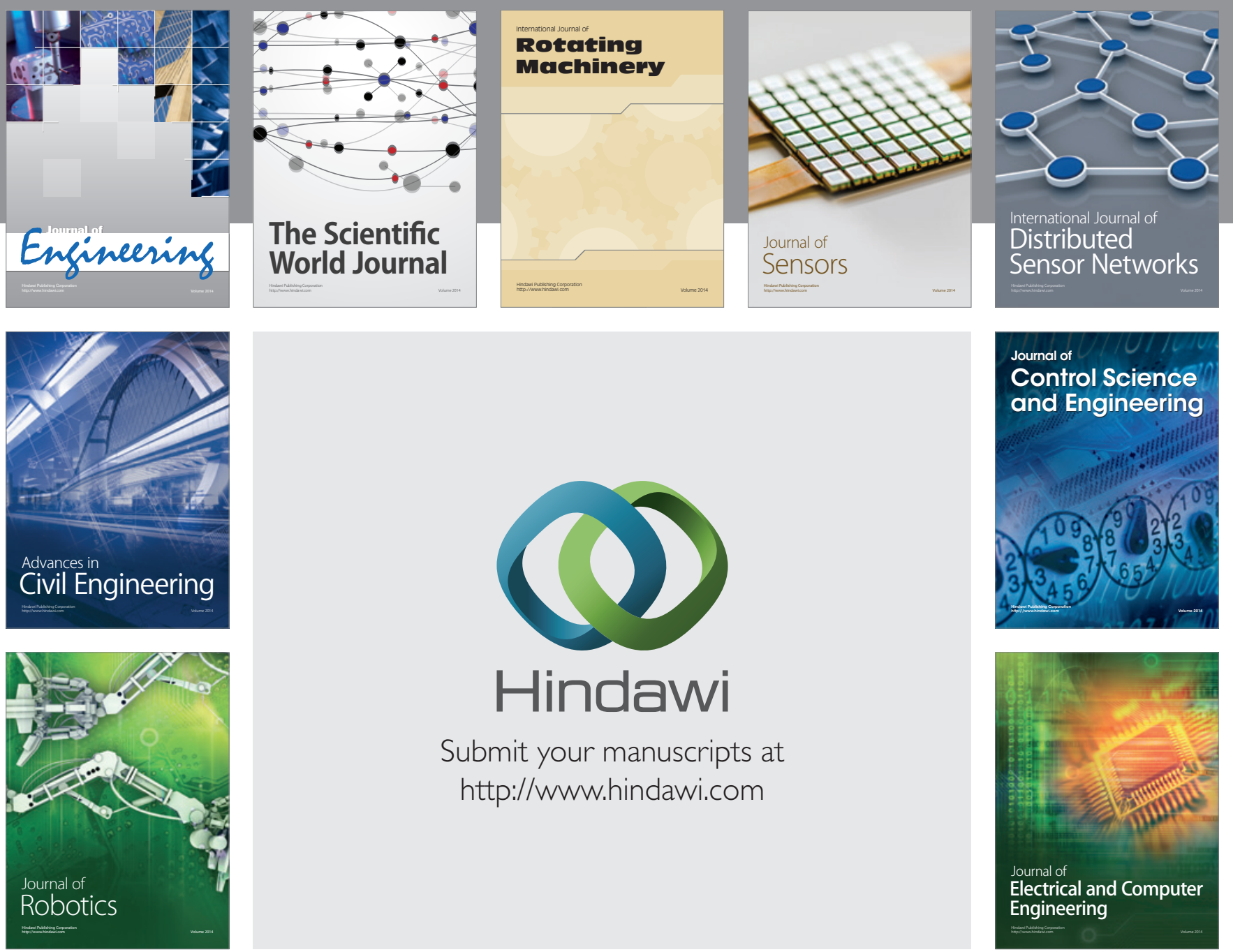

Submit your manuscripts at

http://www.hindawi.com
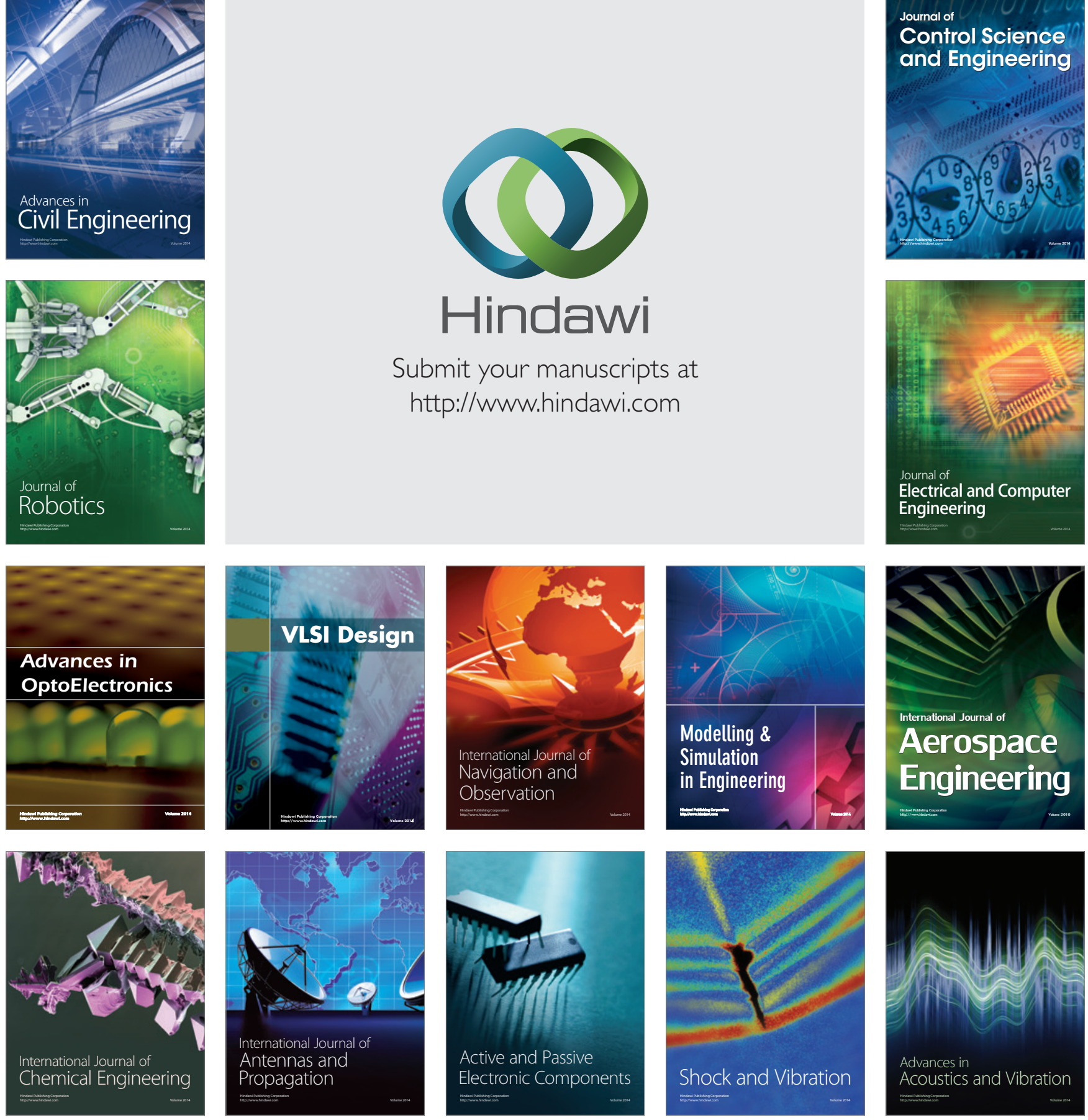